\title{
Is there a cluster in the massive star forming region IRAS $20126+4104$ ?
}

\author{
V. A. Montes ${ }^{1}$, Peter Hofner ${ }^{1,2}$, C. Anderson ${ }^{1,2}$, and V. Rosero ${ }^{1,2}$ \\ ${ }^{1}$ Physics Department, New Mexico Tech \\ 801 Leroy Pl., Socorro, NM 87801, USA \\ email: vmontes@nmt.edu \\ ${ }^{2}$ National Radio Astronomy Observatory, \\ 1003 Lopezville Road, Socorro, NM 87801, USA
}

\begin{abstract}
A Chandra X-ray Observatory ACIS-I observation and a $6 \mathrm{~cm}$ continuum radio observation with the Karl G. Jansky Very Large Array (VLA) together with a multiwavelength study in infrared (2MASS and Spitzer) and optical (USNO-B1.0) shows an increasing surface density of X-ray sources toward the massive protostar. There are at least 43 YSOs within $1.2 \mathrm{pc}$ distance from the massive protostar. This number is consistent with typical B-type stars clusters (Lada \& Lada 2003).
\end{abstract}

Keywords. Stars: formation, X-ray: stars, Infrared: stars, radio continuum: stars

\section{Introduction}

Two main competitive theories intend to explain massive star formation: the turbulent core model, which is an extension of the low-mass star formation model (McKee \& Tan 2003), and models involving competitive accretion or stellar collisions (Bonnell \& Bate 2006). The characterization of the cluster in which massive stars form can help discriminate between the two main scenarios. Until recently it was believed that massive stars were only formed in substantial clusters. However, a recent study of the massive star forming region IRAS 20126+4104 using Spitzer observations by Qiu et al. (2008), suggested that the massive protostar was isolated, and the region was showing no obvious cluster. This is a well studied massive protostar located at a distance of $1.7 \mathrm{kpc}$. This early B-type star is currently forming through disk accretion (Cesaroni et al. 2014), has a luminosity of about $10^{4} \mathrm{~L}_{\odot}$, and is associated with a bipolar outflow (Cesaroni et al. 1997, Lebron et al. 2006). Here we adopt a multiwavelength approach to characterize the stellar environment of the IRAS 20126+4104 region combining Chandra X-ray ACIS-I and VLA $6 \mathrm{~cm}$ continuum observations with near-infrared (2MASS) data of the region. We detected $150 \mathrm{X}$-ray sources in the ACIS-I field and 13 radio sources within the $9^{\prime} .2$ VLA primary beam. Associating X-ray sources with their near-infrared counterparts in the 2MASS catalog, and a color study of those counterparts, allow us to determine the foreground/background contamination, and to investigate the population of young stars in the IRAS $20126+4104$ region.

\section{Analysis: Contamination and source characteristics}

The analysis of the region explicitly explained in Montes et al. (2015), was mainly done using the 2MASS counterparts of the X-ray sources. It was found that $88 \mathrm{X}$-ray sources have near-infrared counterparts and consequently 62 have no 2MASS counterparts. Plotting the positions of the latter sources shows a uniform distribution over 
the ACIS-I field contrasting with the positional plot of the X-ray sources having nearinfrared counterparts which appear clustered around the protostar. The distribution of the sources without 2MASS counterparts is consistent with extragalactic contamination due to AGNs (20 \pm 10 sources from Getman et al. (2006) simulation) and late-type stars which would be below the 2MASS sensitivity limit at this distance. From the 88 MASS counterparts, 79 with good photometry quality were used to plot the $\mathrm{H}_{-} \mathrm{K}_{s}$ versus $\mathrm{J}-\mathrm{H}$ color-color diagram showing that the majority of the sources appear to be located in the color space corresponding to WTTS, 3 are in the CTTS locus and one having a large infrared excess which is likely a class I protostar. The 79 counterparts were also used to plot the J versus J-H color-magnitude diagram and compared to the Besançon simulation of Galactic stellar populations (Robin et al. 2003) allowing to determine the location of expected foreground and background contamination in the J versus J-H color-magnitude diagram which results in 30 sources. A similar study with optical data using USNO-B1.0 catalog plotting the $\mathrm{R}$ versus $\mathrm{R}-\mathrm{I}$ color-magnitude diagram allows to add 10 more sources to the contamination list of foreground and background sources. This study determines that approximatively $80 \mathrm{X}$-ray sources are associated with the massive protostar.

\section{Discussion}

In the previous study of the cluster by Qiu et al. (2008), an area of 5'x 5' was observed with the Spitzer Space Telescope and 19 YSOs were identified, leading to the conclusion that no obvious cluster is associated with the massive protostar. In our study, $23 \mathrm{X}$-ray sources were identified in the same area, with only 3 sources coincident with the sources found by Qiu et al. (2008). We also find 7 compact radio sources in this area, with 4 of them having neither X-ray nor Spitzer counterparts. Thus, combining the results of our study and that of Qiu et al. (2008), we find 43 YSOs within 1.2 pc radius from the massive protostar. This is likely a lower limit. Comparing it with the compilation of clusters in Lada \& Lada (2003, Table 1), this number is consistent with typical embedded clusters about B-type stars. Another hint for the presence of a cluster is the increase of the surface density of X-ray sources toward the massive protostar (Montes et al. 2015). In conclusion, the massive protostar IRAS $20126+4104$ is associated with about 80 X-ray sources, which are likely lower mass YSOs.

\section{Acknowledgement}

P. H. acknowledges support from NSF grant AST-0908901 and CXC award G03-4018A.

\section{References}

Bonnell, I. A. \& Bate, M. R. 2006, MNRAS, 370, 488

Cesaroni, R., Felli, M., Testi, L., Walmsley, C. M., \& Olmi, L. 1997, A\&A, 325, 725

Cesaroni, R., Galli, D., Neri, R., \& Walmsley, C. M. 2014, A\&AA, 566, A73

Getman, K. V., Feigelson, E. D., Townsley, L., Broos, P., Garmire, G., \& Masahiro Tsujimoto, M. 2006, ApJS, 163, 306

Lada, C. J., Lada E. A. 2003, ARA $\mathscr{G} A, 41,57$

Lebron, M., Beuther, H., Schilke, P., \& Stanke, Th. 2006, A\&A, 448, 1037

Mckee, C. F. \& Tan, J. C. 2003, ApJ, 585, 850

Montes, V. A., Hofner, P., Anderson, C., \& Rosero, V. 2006, ApJS, 219, 41

Robin, A. C., Reyl, C., Derrire, S., \& Picaud, S. 2003, A\&A, 409, 523

Qiu, K., Zhang, Q., Megeath, T., Gutermuth, R. A., Beuther, H., Shepherd, D. S., Sridharan, T. K., Testi, L., \& De Pree, C. G. 2008, ApJ, 685, 1005 